\title{
YOICHIRO NAMBU
}

Date of birth:

Birthplace:

U. S. Citizenship:
18 January 1921

Tokyo, Japan

31 March 1970

\section{Education}

B. S.

D. Sc.

\section{Positions}

1950-1956

1952-1954

$1954-1956$

1956-1958

1958-1990

1991-
University of Tokyo 1942

University of Tokyo 1952
Osaka City University - Professor

Institute for Avdanced Study - Member

University of Chicago - Research Associate

University of Chicago - Associate Professor

University of Chicago - Professor

Professor Emeritus, University of Chicago 


\section{Honors}

Dannie Heinemann Prize for Mathematical Physics, 1970

Member of National Academy of Sciences, 1971

Member of American Academy of Arts and Sciences, 1971

J. Robert Oppenheimer Prize, 1976

Order of Culture, Government of Japan, 1978

United States National Medal of Science, 1982

Honorary Member of Japan Academy, 1984

Max Planck Medal, 1985

P.A.M. Dirac Medal, ICTP, Trieste, 1986

Wolf Prize, Government of Israel, 1995 


\section{List of Publications}

1948

1. On the Relativistic Formulation of the Perturbation Theory, Prog. Theor. Phys. 3, 444.

2. The Level Shift and the Anomalous Magnetic Moment of the Electron, Prog. Theor. Phys. 4, 82.

3. Second Configuration Space and Third Quantization, Prog. Theor. Phys. 4, 96.

4. Effect of the C-Meson Field on the Anomalous Magnetic Moment of the Electron (with Z. Koba and T. Tati), Prog. Theor. Phys. 4, 99.

5. On the Method of Third Quantization, I and II, Prog. Theor. Phys. 4, 331; 339.

1950

6. A Note on the Eigenvalue Problem in Crystal Statistics, Prog. Theor. Phys. 5, 1.

7. The Use of the Proper Time in Quantum Electrodynamics I Prog. Theor. Phys. 5, 82.

8. On the Electromagnetic Properties of Mesons (with T. Kinoshita), Prog. Theor. Phys. 5, 307.

9. Derivation of the Interaction Potential from Field Theory, Prog. Theor. Phys. 5, 321. 
10. Force Potentials in Quantum Field Theory, Prog. Theor. Phys. 5, 614.

11. On the Interaction of Mesons with the Electromagnetic Field II (with T. Kinoshita), Prog. Theor. Phys. 5, 749.

1951

12. On the Nature of V-Particles I and II (with K. Nishijima and Y. Yamaguchi), Prog. Theor. Phys. 6, 615; 619.

13. Meson-Nucleon Scattering (with Y. Yamaguchi), Prog. Theor. Phys. 6, 1000.

\section{2}

14. On Lagrangian and Hamiltonian Formalism, Prog. Theor. Phys. 7, 131.

15. An Empirical Mass Spectrum of Elementary Particles, Prog. Theor. Phys. 7, 595.

1954

16. The Collective Description of Many-Particle System (A Generalized Theory of Hartree Fields) (with T. Kinoshita),

Phys. Rev. 94, 598.

1955

17. Structure of the Scattering Matrix, Phys. Rev. 98, 803.

18. Structure of Green's Function in Quantum Field Theory I, Phys. Rev. 100, 394.

\section{6}

19. Structure of Green's Function in Quantum Field Theory II, Phys. Rev. 101, 459.

20. Renormalization Constants, Phys. Rev. 101, 1183.

21. Application of Dispersion Relations to Low Energy Meson-Nucleon Scattering (with G.F. Chew, M.L. Goldberger and F.E. Low),

Phys. Rev. 106, 1337. 
22. Relativistic Dispersion Relation Approach to Photomeson Production (with G.F. Chew, M.L. Goldberger and F.E. Low),

Phys. Rev. 106, 1345.

1957

23. Possible Existence of a Heavy Neutral Meson, Phys. Rev. 106, 1366.

24. Dispersion Relations for Nucleon-Nucleon Scattering (with M.L. Goldberger and R. Oehme), Ann. Phys. 2, 226.

25. Parametric Representations of General Green's Functions, Il Nuovo Cimento $X$ 6, 1064.

1958

26. Dispersion Relations for Form Factors, Il Nuovo Cimento $X \mathbf{9}, 610$.

27. Dispersion Theory Treatment of Pion Production in Electron-Nucleon Collisions (with S. Fubini and V. Wataghin), Phys. Rev. 111, 329.

1960

28. Quasi-Particles and Gauge Invariance in the Theory of Superconductivity, Phys. Rev. 117, 648.

29. Axial Vector Current Conservation in Weak Interactions, Phys. Rev. Lett. 4, 380.

30. Electro-production of pi-Mesons (with R. Blankenbecler, S. Gartenhous and R. Hugg), Il Nuovo Cimento $X 17,775$.

31. Anomalous Thresholds in Dispersion Theory I (with R. Blankenbecler), Il Nuovo Cimento $X$ 18, 595.

1961

32. Odd $\Lambda \Sigma$ Parity and the Nature of the $\pi \Lambda \Sigma$ Coupling (with J.J. Sakurai), Phys. Rev. Lett. 6, 377.

33. A 'Superconductor' Model of Elementary Particles and Its Consequences, in the Proceedings of the Midwest Conference on Theoretical Physics, eds. F.J. Belinfante, S.G. Garten haus and R.W. King, Purdue University, Lafayette, Indiana, April 1-2, 1960, p. 1 . 
34. Dynamical Model of Elementary Particles based on an Analogy with Superconductivity I (with G. Jona-Lasinio),

Phys. Rev. 122, 345.

35. Dynamical Model of Elementary Particles based on an Analogy with Superconductivity II (with G. Jona-Lasinio),

Phys. Rev. 124, 246.

36. Possible Bound $\Sigma-\Lambda$ System, (with E. Shrauner), Il Nuovo Cimento X 21, 864 .

1962

37. Chirality Conservation with Soft Pion Production (with D. Lurié), Phys. Rev. 125, 1429.

38. Soft Pion Emission Induced by Electromagnetic and Weak Interactions (with E. Shrauner), Phys. Rev. 128, 862 .

39. Magnetic Field Dependence of the Energy Gap in Superconductors (with San Fu Tuan),

Phys. Rev. 128, 2622.

40. Rare Decay Modes of the $\omega(\eta)$ Meson (with J.J. Sakurai), Phys. Rev. Lett. 8, 79.

1963

41. Double Phase Representation of Analytic Functions (with M. Sugawara), Phys. Rev. 131, 2335.

42. High-Energy Behavior of Total Cross-Sections (with M. Sugawara), Phys. Rev. 132, 2724.

43. Magnetic Field and Phase Transition in Thin Film Superconductors (with San Fu Tuan), Phys. Rev. Lett. 11, 119.

44. Non-shrinking Diffraction Scattering (with M. Sugawara), Phys. Rev. Lett. 10, 304.

45. $\mathrm{K}^{*}(725)$ and the Strangeness-changing Currents of Unitary Symmetry (with J.J. Sakurai), Phys. Rev. Lett. 11, 42. 
46. Self-Consistent Models of Strong interactions with Chiral Symmetry (with P. Pascual), Il Nuovo Cimento $X \mathbf{3 0}, 354$.

\section{4}

47. Considerations on the Magnetic Field Problem in Superconducting Thin Films (with San Fu Tuan),

Phys. Rev. 133, A1.

48. Axial Vector Mesons (with P. Freund), Phys. Lett. 12, 248.

49. Broken $\mathrm{SU}(x) \times \mathrm{SU}(3) \times \mathrm{SU}(3) \times \mathrm{SU}(3)$ Symmetry of Strong Interactions (with P. Freund), Phys. Rev. Lett. 12, 714.

50. Mass and Coupling Constant Formulas in Broken Symmetry Schemes (with P. Freund), Phys. Rev. Lett. 13, 221.

51. Magnetic Field and Phase Transition in Superconducting Thin Films (with San Fu Tuan), Rev. Mod. Phys. 36, 288.

52. Quasi-elementary Massless Bosons Associated with the Quantum Electrodynamics of Johnson, Baker and Willey, Phys. Lett. 9, 214.

53. Outlook of Elementary Particle Physics, in Nature of Matter, ed. Luke C.L. Huan, BNL F88(T-360), p. 55.

1965

54. Three-Triplet Model with Double SU(3) Symmetry (with M.-Y. Han), Phys. Rev. B139, 1006.

55. Dynamical Symmetries and Fundamental Fields, in the Proceedings of Coral Gables Conference 1965, eds. B. Kursunoglu, A. Perlmutter and I. Sakmar (W. H. Freeman and Co.), p. 274.

56. Broken $\mathrm{SU}(3) \times \mathrm{SU}(3) \times \mathrm{SU}(3) \times \mathrm{SU}(3)$ Symmetry (with $\mathrm{P}$. Freund), Ann. Phys. 32, 201.

57. Triplets, Static SU(6), and Spontaneously Broken Chiral SU(3) Symmetry, in Proceedings of the International Conference on Elementary Particles, in Commemoration of the Thirtieth Anniversary of Meson Theory, Kyoto, 1965, ed. Y. Tanikawa (Kyoto Publication Office) p. 131; General Discussion, ibid., p. 327. 
58. Axial Vector Meson and the Hyperfine Structure of Hydrogen (with S. Fenster and R. Koberle), Phys. Lett. 19, 513.

59. Baryon Structure and Electromagnetic Properties, (with S. Fenster), Prog. Theor. Phys. Suppl., Extra No., 250.

60. Electromagnetic Properties of the Baryon (Hyperfine Structure of Hydrogen), 1965 Tokyo Summer Lecture, Part II, ed. G. Takeda (Syokabō Publishing), p. 87.

\section{6}

61. A Systematics of Hadrons in Subnuclear Physics, in Preludes in Theoretical Physics, eds. A. De-Shalit, H. Feshbach and L. van Hove (North-Holland), p. 133.

62. Coupling Constant Relations for $1^{ \pm}$and Induced $0^{ \pm}$Mesons (with J. Cronin), Il Nuovo Cimento A41, 380.

63. Nonleptonic Decays of K-Mesons (with Y. Hara), Phys. Rev. Lett. 16, 875.

64. Nonleptonic Decays of Hyperons (with Y. Hara and J. Schechter), Phys. Rev. Lett. 16, 380.

65. Relativistic Wave Equations for Particles with Internal Structure and Mass Spectrum, Prog. Theor. Phys. Supp. 37 and 38, 368.

\section{7}

66. Infinite Multiplets, in the Proceedings of 1967 International Conference on Particles and Fields, eds. C.R. Hagen et al. (Interscience), p. 347.

67. Infinite Component Wave Equations with Hydrogenlike Mass Spectra, Phys. Rev. 160, 1177.

\section{8}

68. Magnetic Moments and Charge Radii for States Described by an Infinite Component Wave Equation (with S.P. Rosen),

Prog. Theor. Phys. 40, 5.

69. Quantum Electrodynamics in Nonlinear Gauge, Prog. Theor. Phys. Suppl., Extra No., 190.

70. Scalar Fields Coupled to the Trace of the Energy Momentum Tensor (with P. Freund), Phys. Rev. 174, 1741. 
71. $S$-Matrix in Semiclassical Approximation, Phys. Lett. B26, 626.

72. Relativistic Groups and Infinite Component Fields, talk at Nobel Symposium on Elementary Particle Theory, Goteborg, May, 1968, ed. N. Svaartholm (Almquist and Wiksell), p. 105.

\section{0}

73. Asymptotic Behavior of Partial Widths in the Veneziano Model of Scattering Amplitudes (with Paul Frampton), in QUANTA, A Collection of Scientific Essays dedicated to Gregor Wentzel, eds. P.G.O. Freund, C. Goebel and Y. Nambu (University of Chicago Press), p. 33.

74. Quark Model and the Factorization of the Veneziano Amplitude, in the Proceedings of the International Conference on Symmetries and Quark Models, Wayne University, 1969, ed. R. Chand (Gordon and Breach), p. 269.

75. Statistical Approach to the Veneziano Model (with L.-N. Chang and P. Freund), Phys. Rev. Lett. 24, 628.

76. Axial-Vector Form Factor of Nucleon Determined from Threshold Electropion Production (with M. Yoshimura),

Phys. Rev. Lett. 24, 25.

77. Symmetry Breakdown and Small Mass Bosons,

Fields and Quanta 1, 33 (1970), also published in The Past Decade in Particle Theory, eds. E.C.G. Sudarshan and Y. Ne'eman (Gordon and Breach, 1973), p. 33.

78. Duality and Hadrodynamics, notes prepared for Copenhagen High Energy Symposium, August 1970 (unpublished).

1971

79. Electromagnetic Currents in Dual Hadrodynamics, Phys. Rev. D4, 1195.

\section{2}

80. Gauge Conditions in Dual Resonance Models (with F. Mansouri), Phys. Lett. B39, 375.

81. Use of Regulator Fields in Dual Resonance Models (with J. Willemsen), submitted to XVI International Conference on High Energy Physics, 1972, eds. J.D. Jackson and A. Roberts, National Accelerator Laboratory, 1972, number 110, unpublished.

82. Chiral Symmetries and Current Algebras, Lecture at International Summer School, Erice, Italy, 1972 (unpublished). 
83. Generalized Hamiltonian Dynamics, Phys. Rev. D7, 2405.

84. Models Concerning the Chemical Structure of Hadrons, in the Proceedings of Tokyo Symposium on High Energy Physics, July.

85. Elementary Particle Physics in Perspective, Butsuri 28, 452 (in Japanese).

1974

86. Three Triplets, Paraquarks and "Colored" Quarks (with M.-Y. Han), Phys. Rev. D10, 675.

87. Quarks, Strings and Gauge Fields, in the Proceedings of Johns Hopkins Workshop on Current Problems in High Energy Particle Theory, 1973.

88. Strings, Monopoles and Gauge Fields, Phys. Rev. D10, 4262.

\section{5}

89. Dynamics of the Zweig-Iizuka Rule and a New Vector Meson Below $2 \mathrm{GeV} / \mathrm{c}^{2}$ (with P. Freund),

Phys. Rev. Lett. 34, 1645.

1976

90. Magnetic and Electric Confinement of Quarks, talks given at Topical Conference on Extended Systems, Ecole Normale Superieure, June, 1975; Phys. Rep. C23, 250.

91. Diquark Color Excitation and the Narrow Resonances (with M.-Y. Han), Phys. Rev. D14, 1459.

1977

92. Strings, Vortices and Gauge Fields, in Quark Confinement and Field Theory, eds. D.R. Stump and D.H. Weingarten (John Wiley and Sons), p. 1.

93. Description of Hadronic Structure, in the Proceedings of the International School on Elecrtro- and Photonuclear Reactions, 1976, eds. S. Costa and C. Shaerf; also published in Quark Models and Hadronic Structure, Physics Workshop Series, 1, ed. G. Morpurgo (Plenum Press), p. 281.

94. Monopoles, Strings and Instantons, in Five Decades of Weak Interactions, Proceedings of CCNY Conference, 1977, ed. N. P. Chang Ann. of the N.Y. Acad. of Sci. 74, 294. 
95. Elementary Particles, Butsuri 32, 11 (in Japanese).

96. String-Like Configurations in the Weinberg-Salam Theory, Nucl. Phys. B130, 505.

\section{8}

97. Some Topological Configurations in Gauge Theories, Int. J. Theor. Phys. 17, 287.

98. Remarks on the Topology of Gauge Fields, in New Frontiers in High Energy Physics, Proceedings of the Orbis Scientiae, University of Miami, January 1978, eds. Kursunoglu and Perlmutter (Plenum Press), p. 632.

\section{9}

99. Topological Problems in Gauge Theories, talk at UCLA Symposium in Honor of Julian Schwinger on the Occasion of His 60th Birthday, Physica A96, 89.

100. QCD and the String Model, Phys. Lett. B80, 372.

101. Concluding talk at the XIX International Conference on High Energy Physics, Tokyo, 1978; in the Proceedings of the Conference, eds. S. Homma et al. (Phys. Soc. Japan), p. 971 .

1980

102. Quark Confinement: the Cases for and Against, in To Fulfill a Vision: Jerusalem Einstein Centennial Symposium on Gauge Theories and Unification of Physical Forces, ed. Y. Ne'eman (Addison and Wesley), p. 118.

103. Hamilton-Jacobi Formalism for Strings, Phys. Lett. B92, 327.

104. Effective Abelian Gauge Fields, Phys. Lett. B102, 149.

105. Strings and Vortices,

VPI Conference on Weak Interactions as Probes of Unification, AIP Conference Proceedings, Particles and Fields subseries No. 23, p. 633.

106. Quark (Kodansha Publishers) in Japanese.

107. Theory of Strings, in the Proceedings of the 1981 INS Symposium on Quark and Lepton Physics, Tokyo, Japan, eds. K. Fujikawa et al. (Institute of Nuclear Study, Univ. of Tokyo), p. 347. 
1982

108. Many Quark Problem in Two Dimensional Gauge Theory (with B. Bambah), Phys. Rev. D26, 2871.

109. One-Dimensional Quark Gas (with B. Bambah and M. Gross), Phys. Rev. D26, 2875.

1983

110. Concluding Remarks, Lecture Notes in Physics, 176; Proceedings of the International Symposium on Gauge Theory and Gravitation, Nara, Japan, 1982, eds. K. Kikkawa et al., (Springer), p. 277.

111. Magnetic Monopoles and Related Topics, in the Proceedings of the Topical Conference, June, 1982, eds. T. Eguchi and Y. Yamaguchi (World Scientific), p. 3.

112. The Activity of the Tomonaga Group up to the Time of the 1947 Shelter Island Conference (with K. Nishijima), in Shelter Island II, Proc. of the 1983 Shelter Island Conference on Quantum Field Theory and the Fundamental Problems of Physics, eds. R. Jackiw et al. (MIT Press), p. 367.

\section{4}

113. Concluding Remarks, in the Proceedings of the Solvay Conference on Higher Energy Physics, Austin, Texas, November 1982; Phys. Rep. 104, 237.

114. Quantum Mechanics: Prospects and Problems, in the Proceedings of the Symposium on Foundations of Quantum Mechanics in Light of New Technology, Tokyo, August, 1983, eds. Kamefuchi et al. (Physical Society of Japan), p. 363.

115. Superconductivity and Particle Physics, talk at International Conference on Low Temperature Physics, Karlsruhe, Germany, August, 1984; Physica B126, 328.

116. The Turbulent Ether, Symmetries in Particle Physics, Symposium in Honor of F. Gürsey's 60th Birhtday, eds. I. Bars, A. Chodos and C.-H. Tze (Plenum Press), p. 9.

117. Fermions Living in a Space of Lie Groups, in From SU(3) to Gravity, Festschrift in Honor of Yuval Ne'eman, eds. E. Gotzman and G. Tauber (Cambridge Univ. Press), p. 45.

1985

118. Topological Excitations in Physics, Lectures in Applied Mathematics, 21, 3; (Proceedings of 1982 Mathematical Seminar, American Mathematical Society). 
119. Fermion-Boson Relations in BCS-Type Theories, in Supersymmetry in Physics, Proceedings of the Los Alamos Workshop on Supersymmetry in Physics, December, 1983, eds. A. Kosteleck'y and D.K. Campbell (North-Holland); also published as Physica D15, 147.

120. Some Theoretical Problems in Particle Physics, Phys. Blätter 41, 173.

121. Directions of Particle Physics, Proceedings of the Kyoto International Symposium, MESON50, 1985, eds. M. Bando, R. Kawabe and N. Nakanishi, Prog. Theor. Phys. Suppl. 85, 104.

122. Gauge Principles, Vector Meson Dominance and Spontaneous Symmetry Breaking, International Symposium on Particle Physics in the 1950s, Fermilab, May.

\section{6}

123. Supersymmetry and Superconductivity, in Rationale of Beings, Festschrift in Honor of Gyo Takeda, eds. K. Ishikawa et al. (World Scientific), p. 3.

\section{7}

124. Field Theory of Galois Fields, in Field Theory and Quantum Statistics, Essays in Honor of the Sixtieth Birthday of E.S. Fradkin, eds. I.A. Batalin et al. (Adam Hilgar), p. 625 .

125. Thermodynamic Analogy in Quantum Field Theory, in Wanderings in Physics, Festschrift in Honor of K. Nishijima, eds. K. Kawarabayashi and A. Ukawa (World Scientific), p. 167.

\section{8}

126. BSC Mechanism and the Interacting Boson Model (with M. Mukherjee), Phys. Lett. B209, 1.

127. Dynamical Symmetry Breaking in Nuclei, in Festi-Val, Festschrift in Honor of Valentine Telegdi's 60th Birthday, ed. K. Winter (North-Holland), p. 181.

128. BCS Theory, Quasi-Supersymmetry and Nuclear Physics, (Encuentro LatinoAmericano de Fisica de Alta Energia, Universidad Federal Santa Maria, Valparaiso, Chile, December 1987, eds. N. Bralic et al., Universidad Fedederal Santa Maria), Sciencia 165-166, 141.

129. The BCS Theory and the Sigma Model Revisited, in Themes in Particle Physics, dedicated to 70th Birthday of Julian Schwinger, eds. S. Deser and R.J. Finkelstein (World Scientific). 
130. BCS Mechanism, Quasi-Supersymmetry and Fermion Masses, in the Proceedings of XI Warsaw Symposium on Elementary Particle Physics, New Theories in Physics, Kazimierz, Poland, May 1988, eds. Z. Ajduk et al. (World Scientific), p. 1.

131. Quasi-Supersymmetry, Bootstrap Symmetry Breaking and Fermion Masses, in the Proceedings of the 1988 International Workshop on New Trends in Strong Coupling Gauge Theories, Nagoya, August 1988 (World Scientific), p. 3.

132. BCS and IBM (with M. Mukherjee), Ann. Phys. 191, 143.

1990

133. Particle Physics since Lawrence and Yukawa, Particle Acclerators, 26 p. 89: Proceedings of the 14th International Conference on High Energy Accelerators, Part I, eds. Y. Kimura et al. (Gordon and Breach).

134. Model Building Based on Bootstrap Symmetry Breaking, in the Proceedings of the 1989 Workshop on Dynamical Symmetry Breaking, Nagoya, December 1989, eds. T. Muta and K. Yamawaki (Nagoya University), p. 1.

135. Mass Formulas and Dynamical Symmetry Breaking, in From Symmetries to Strings: Forty Years of Rochester Conferences, A Symposium to Honor Susumu Okubo on His 60th Year, May 1990, ed. A. Das (World Scientific), p. 1.

136. New Bootstrap and the Standard Model, Plots, Quarks $\mathcal{G}$ Strange Particles, Proceedings of the Dalitz Conference, Oxford, July 1990, eds. I.J.R. Aitchison, C.H. Llewellyn Smith and J.E. Paton (World Scientific), p. 56.

137. More on Bootstrap Symmetry Breaking, in the Proceedings of the 1990 International Workshop on Strong Coupling Gauge Theories and Beyond, Nagoya, Japan, July 1990, eds. T. Muta and K. Yamawaki (World Scientific), p. 3.

138. Summary of Personal Recollections of the Tokyo Group, Elementary Particle Theory in Japan, 1935-1960 - Japan-US Collaboration on the History of Particle Physics (Lake Yamanaka, 1985), eds. L. Brown et al., Prog. Theor. Phys. Suppl. 105, 111.

1991

139. Supersymmetry and Quasi-supersymmetry, in Elementary Particles and the Universe, Essays in Honor of Murray Gell-Mann, ed. J.H. Schwarz (Cambridge Univ. Press), p. 89 .

140. Theoretical Perspective in High Energy Physics, in Proceedings of the 25th International Conference on High Energy Physics, Singapore, eds. K.K. Phua and Y. Yamaguchi (South East Theoretical Association), p. 51. 
141. Dynamical Symmetry Breaking, in Evolutionary Trends in the Physical Sciences, Proceedings of the Yoshio Nishina Centennial Symposium, Tokyo, Japan, December 1990, eds. M. Suzuki and R. Kubo (Springer-Verlag), p. 51.

142. Majorana's Infinite Component Wave Equation, A Commentary to be published in the Collected Papers of E. Majorana, ed. V. Telegdi.

143. Old Wine in New Bottles - Mass Spectra, Bootstrap and Hierarchy, talk given at E. C. G. Sudarshan's Contributions to Theoretical Physics, ed. A. Gleeson.

\section{2}

144. Dynamical Symmetry Breaking: An Overview, in the Proceedings of the International Workshop on Electroweak Symmetry Breaking, Hiroshima, Japan, November, 1991, eds. W.A. Bardeen et al. (World Scientific).

145. Cosmic Rays and Particle Physics, talk at the International Workshop on Super-high Energy Hadronic Interactions at Waseda University, October 1991.

\section{3}

146. Spontaneous Symmetry Breaking and the Origin of Mass, in Some New Trends on Fluid Mechanics and Theoretical Physics, in Honor of Professor Pei-Yuan Chou's 90th Anniversary, 1992, eds. C.C. Lin and Ning Hu (Peking University Press), p. 97.

147. Nonlocal Separable Solutions of the Inverse Scattering Problem (with T. Gherghetta), Int. J. Mod. Phys. A8, 3163.

148. Feedback Effects in Superconductors (with T. Gherghetta), Phys. Rev. B49, 49. 